\section{PREPRINT WATCH}

\section{Super(antigen) target for SARS-CoV-2}

In the later stages of COVID-19, SARS-CoV-2 proceeds to infect the lower respiratory tract, causing viral pneumonitis and, in severe cases, systemic coagulopathy and hyperinflammation. This hyperinflammatory state has similarities to the toxic shock syndrome caused by superantigens (SAgs). SAgs, which are usually found in unprocessed bacterial toxins, bind directly to an MHC molecule and one of the variable domains of the T cell receptor (TCR), and this cross-linking leads to an excessive release of inflammatory cytokines. Previous in silico analyses have identified a SAg motif adjacent to the S1-S2 cleavage site of SARS-CoV-2 spike (S) glycoprotein, which has a sequence and structure similar to staphylococcal enterotoxin $B$ (SEB). In addition, TCR V $\beta$ skewing, which is indicative of SAg-mediated T cell activation, has been observed in patients with COVID-19 who have severe hyperinflammation.

This preprint (non-peer-reviewed) by Cheng et al. further characterizes the SAg motif in SARS-CoV-2 $S$ protein and evaluates a monoclonal antibody capable of targeting this region. Molecular docking simulations confirmed that the SAg motif of S protein fits into the catalytic cavities of transmembrane protease serine 2 (TMPRSS2) and furin, which stabilizes S before S1-S2 cleavage. Given the homology of this motif with SEB, antibodies to SEB were screened in silico for their ability to bind the SAg motif on S protein. The monoclonal antibody $6 \mathrm{D} 3$ engaged $\mathrm{S}$ with a binding affinity comparable to that of TMPRSS2 and furin and was capable of inhibiting infection with live SARS-CoV-2, as assessed by immunofluorescent staining of double-stranded RNA and $\mathrm{S}$ in Vero-E6 cells.

Most SARS-CoV-2 neutralizing antibodies isolated from convalescent patients have been identified using recombinant S proteins with a mutated S1-S2 cleavage site. This helps to stabilize the $\mathrm{S}$ trimer but fails to capture antibodies that bind to the cleavage site and potentially the adjacent SAg motif. Viral challenge studies in vivo will be necessary for the clinical translation of SAg-targeting antibodies. Furthermore, ex vivo stimulation of patient T cells with SARS-CoV-2 S protein and SEB will be needed to confirm the possibility of SAg-mediated T cell activation in patients with severe COVID-19. Nonetheless, monoclonal antibodies targeting the $S$ protein cleavage site are potentially capable of virus neutralization and mitigation of shock following infection with SARS-CoV-2 and other human coronaviruses that encode furin-like cleavage sites, specifically MERS, HKU1 and OC43.

Matthew Brown and Nina Bhardwaj OxMS Preprint Journal Club, Icahn School of Medicine at Mount Sinai, New York, NY, USA

e-mail: highlights@preprintclub.com ORIGINAL ARTICLE Cheng, M. H. et al. A monoclonal antibody against staphylococcal enterotoxin B superantigen inhibits SARS-CoV-2 entry in vitro. Preprint at bioRxiv https://doi.org/10.1101/2020.11.24.395079 (2020) RELATED ARTICLE Brown, M. Oxford-Mount Sinai (OxMS) Preprint Journal Club. OxMS https://www.preprintclub.com/2021-jan-cheng (2020)

\title{
mRNA vaccine shows promise in autoimmunity
}

The ideal treatment for an autoimmune disease should specifically target autoreactive cells, without the need for systemic immune suppression. Various approaches to antigen-specific tolerization of autoreactive T cells have been studied, with limited success. Now, Ugur Sahin and colleagues demonstrate that mRNA-based vaccines not only hold promise for ending the COVID-19 pandemic but can also induce antigen-specific tolerance in mouse models of multiple sclerosis.

The authors had previously developed liposomal formulations of mRNA vaccines (mRNA-LPX) that are optimized for the systemic delivery of mRNA-encoded antigens to lymphoid tissue-resident CD11 $\mathrm{c}^{+}$ antigen-presenting cells (APCs). Normally, mRNA vaccination induces strong $\mathrm{T}$ helper $1\left(\mathrm{~T}_{\mathrm{H}} 1\right)$ responses via the activation of Toll-like receptors (TLRs). However, mRNA that contains 1-methylpseudouridine ( $\mathrm{m} 1 \Psi)$ instead of uracil has strongly reduced inflammatory properties, because the modification abrogates binding to TLR7. The authors hypothesized that vaccination with $\mathrm{m} 1 \Psi$-modified mRNA (m1 $\Psi$ mRNA-LPX) may allow for antigen-presentation by $\mathrm{CD} 11 \mathrm{c}^{+}$ APCs in a non-inflammatory context and thereby induce antigen-specific tolerance.

To test this hypothesis, the authors formulated mRNA, with or without the $\mathrm{m} 1 \Psi$ mutation, with liposomes that lacked adjuvant capacity. Unlike vaccination with mRNA-LPX, m1 $\Psi$ mRNA-LPX did not induce inflammatory cytokines or activate immune cells and allowed for higher and prolonged antigen expression.

Next, the modified mRNA was tested in the experimental autoimmune encephalomyelitis (EAE) model of multiple sclerosis.
In this model, pathology is induced by immunizing mice with a peptide derived from myelin oligodendrocyte glycoprotein $\left(\mathrm{MOG}_{35-55}\right)$. Mice that were vaccinated with $\mathrm{MOG}_{35-55^{-}}$

encoding $\mathrm{m} 1 \Psi$ mRNA on days 7 and 10 after immunization with $\mathrm{MOG}_{35-55}$ were completely protected from disease development. Vaccination of mice with established disease prevented further disease progression and in some cases even reverted pathology.

The authors found that the treatment induced de novo FOXP ${ }^{+}$ regulatory $\mathrm{T}\left(\mathrm{T}_{\mathrm{reg}}\right)$ cells and enhanced the expression of exhaustion markers such as PD1 and CTLA4 on antigen-specific $\mathrm{CD} 4^{+} \mathrm{T}$ cells. Importantly, it was also effective in models of EAE induced with a different protein epitope ( $\left.\mathrm{PLP}_{139-151}\right)$, indicating that the vaccination can induce bystander tolerance. However, it did not affect immune responses to completely unrelated antigens.

A detailed analysis by single-cell sequencing showed that the $\mathrm{m} 1 \Psi$ mRNA vaccine induces the specific suppression of disease-promoting $\mathrm{T}_{\mathrm{H}} 1, \mathrm{~T}_{\mathrm{H}} 17$ and $\mathrm{T}_{\mathrm{H}} 1 / \mathrm{T}_{\mathrm{H}} 17$ cells by effector $\mathrm{T}_{\text {reg }}$ cells, rather than the deletion of autoreactive cells. This suppression appeared to be mediated by co-inhibitory molecules, as treatment with PD1-targeted or CTLA4-targeted checkpoint inhibitors almost completely abolished the protective effect of the vaccine.

The authors point out that the $\mathrm{m} 1 \Psi$ mRNA approach is highly flexible, fast and cost efficient. This may allow for the personalization of the vaccine and the combination of different autoantigens.

Alexandra Flemming

ORIGINAL ARTICLE Krienke, C. et al.

A noninflammatory mRNA vaccine for treatment of experimental autoimmune encephalomyelitis.

Science 371, 145-153 (2021) 